\title{
Pourquoi les jeunes Français ont-ils à 15 ans des compétences inférieures à celles de jeunes d'autres pays?
}

Denis Meuret

Les scores des élèves français à l'évaluation internationale PISA sont seulement moyens. On essaie ici de comprendre pourquoi en comparant la situation de la France et de celle des pays qui la surpassent quant à différentes variables associées à des conditions d'apprentissage efficace. Il apparaît que la France se compare plutôt favorablement à ces pays quant à l'organisation de son système éducatif, quant à l'attitude des familles et des élèves, mais plutôt défavorablement quant aux attitudes des enseignants vis-à-vis de leurs élèves. On essaie ensuite de voir si le système français ne compenserait pas cette efficacité seulement moyenne par un caractère particulièrement équitable. La réponse est plutôt négative.

Mots clés : efficacité, équité, compétences, PISA, comparaisons internationales.

I importe d'être attentif à la place de la France dans les comparaisons internationales des compétences des élèves, non par inclination pour la compétition, mais parce que ce qui y est comparé est la capacité de chaque nation de transmettre à ses enfants des compétences qui leur sont nécessaires pour continuer d'apprendre, pour maîtriser leur propre vie et leur participation à la société.

L'évaluation internationale entreprise sous l'égide de I'OCDE, PISA (Programme for International Student Assessment, cf. annexe 1) a mesuré en 2000 les compétences des élèves de 15 ans en compréhension de l'écrit, mathématiques et sciences dans 32 pays. Ces capacités sont des connaissances mais aussi la capacité de les appliquer à diverses situations de la vie ou de l'apprentissage. II s'agit par exemple de savoir dans quelle mesure les élèves sont capables d'un « usage fonctionnel des connaissances mathématiques, plutôt que de seulement les maîtriser dans le cadre des programmes scolaires » ou encore « la capacité de comprendre, d'utiliser des textes écrits et de réfléchir dessus de façon à atteindre ses buts, à développer ses compétences et son potentiel et de participer à la société (1) ».

En bref, que «la France » fasse moins bien que «le Royaume-Uni » n'est pas grave en soi, mais que les élèves français soient, en termes de compétences, plutôt moins bien armés pour la vie que les élèves de 
ce pays l'est davantage et doit nous interroger. II ne s'agit pas de compétition, mais d'émulation, certainement. Si même un seul pays a atteint un niveau supérieur au nôtre, cela signifie que les conditions sociales et scolaires l'y ont rendu possible. II convient alors d'essayer de comprendre pourquoi, du moins si nous prétendons vouloir le mieux pour les enfants de notre pays.

Les délégations envoyées pour cela dans les pays les plus performants se sont souvent entendu répondre "Nous ne savons pas ". II paraît effectivement plus judicieux de mobiliser à cette fin ce que nous savons des facteurs qui agissent sur l'efficacité de l'enseignement et les données recueillies, dans PISA même, sur le fonctionnement des systèmes éducatifs. C'est ce que l'on voudrait faire dans cet article.

Nous comparerons la situation de la France à celle des pays dont les élèves font, en moyenne, significativement mieux que les élèves français dans au moins deux domaines des trois pris en compte dans PISA: compréhension de l'écrit, mathématiques, sciences (2).

Trois groupes de pays sont dans ce cas. II s'agit de deux pays scandinaves (Finlande et Suède), de deux pays asiatiques (Corée et Japon) et de quatre pays du Commonwealth (Australie, Canada, Nouvelle Zélande et le Royaume-Uni lui-même). En outre, nous étudierons le cas des États-Unis, pays que nous surpassons en maths et dont nos résultats sont voisins en lecture et sciences, mais auquel la France aime généralement à se comparer (3).

On présentera ici les écarts de performance des élèves, puis les variables par lesquelles nous proposons de rendre compte de ces écarts, puis une comparaison avec les pays ci-dessus mentionnés pour ces variables, et enfin nous comparerons les résultats des élèves du point de vue de l'équité, pour voir si ces pays "payent» leur meilleure efficacité d'une inéquité plus grande de leurs systèmes éducatifs.

\section{LES ÉCARTS DE PERFORMANCES MOYENNES DES ÉLĖVES}

Les performances des élèves français dans PISA sont moyennes. En sciences et en compréhension de l'écrit, elles ne sont pas significativement différentes de la moyenne internationale. En maths, elles sont supérieures. Sur 32 pays qui ont participé à cette évaluation - les 28 pays membres de l'OCDE plus le Brésil, la Russie, la Lettonie et le Liechstenstein -,
31 ont été classés, et la France est $14^{\mathrm{e}}$ en compréhension de l'écrit (plus rigoureusement : entre le $11^{\mathrm{e}}$ et le $16^{\mathrm{e}}$ rang, compte tenu des erreurs de mesure), $10^{\mathrm{e}}$ en maths, $12^{\mathrm{e}}$ en sciences.

Ce rang n'a pas été une surprise pour les sciences. II l'a été davantage pour les mathématiques et la compréhension de l'écrit où les évaluations internationales précédentes, conçues par I'IEA - Reading literacy, pour la lecture (1991), SIMSS (1984) et TIMSS (1994) pour maths et sciences - nous avaient habitués à de meilleurs classements.

Cela ne prouve pas que le niveau relatif, a fortiori le niveau absolu, des élèves français a baissé. Les évaluations précédentes mesuraient, elles, la maîtrise de connaissances sur la partie du programme commune aux différents pays (4); en outre, - là est sans doute l'explication principale - elles portaient sur les élèves scolarisés à un certain niveau (la quatrième, le plus souvent) et non sur les élèves d'un âge donné, ce qui nous avantageait par rapport aux pays qui ne pratiquent pas le redoublement. II convient donc d'attendre les prochaines éditions de PISA pour comparer l'évolution relative des compétences d'un pays à l'autre.

En 2000, sur les compétences mesurées dans PISA, comment la France se situe-t-elle par rapport à ces pays?

De fait, les élèves de ces pays ont, en moyenne, des performances significativement plus élevées que celles des élèves français dans les trois domaines, si I'on met à part les USA, bien sûr, et aussi la Suède, qui a des performances plus élevées que la France en compréhension de l'écrit et en sciences, mais inférieures en mathématiques.

Ces écarts sont-ils vraiment importants ? Considérons ceux qui affectent les scores en compréhension de l'écrit, les plus fiables de PISA (sur la fiabilité des données de PISA, cf. annexes 1 et 2). L'écart le plus fort - 41 points avec la Finlande représente $8 \%$ du score moyen de la France. L'écart le plus faible - 11 points avec la Suède - en représente $2 \%$. Une autre façon de prendre la mesure de ces écarts est de les comparer à d'autres: en moyenne, parmi les pays de l'OCDE, 81 points séparent la performance moyenne des élèves appartenant aux quarts haut et bas de l'échelle sociale ; 67 points séparent en moyenne le score des élèves dont la mère a fait des études supérieures et celui des élèves dont la mère a arrêté ses études avant le second cycle du secondaire (5). 
Tableau I. - Les performances moyennes des élèves en compréhension de l'écrit, maths, et sciences

\begin{tabular}{|c|c|c|c|c|c|c|c|c|c|c|c|}
\hline & France & Australie & Canada & $\begin{array}{l}\text { Nouvelle- } \\
\text { Zélande }\end{array}$ & $\begin{array}{c}\text { Royaume- } \\
\text { Uni (1) }\end{array}$ & Suède & Finlande & USA & Corée & Japon & $\begin{array}{c}\text { Moyenne } \\
\text { internationale }\end{array}$ \\
\hline $\begin{array}{l}\text { Compréhension } \\
\text { de l'écrit }\end{array}$ & $\begin{array}{l}505 \\
(2,7)\end{array}$ & $\begin{array}{l}528 \\
(3,5)\end{array}$ & $\begin{array}{c}534 \\
(1,6)\end{array}$ & $\begin{array}{l}529 \\
(2,8)\end{array}$ & $\begin{array}{l}523 \\
(2,6)\end{array}$ & $\begin{array}{l}516 \\
(2,2)\end{array}$ & $\begin{array}{l}546 \\
(2,6)\end{array}$ & $\begin{array}{l}504 \\
(7,0)\end{array}$ & $\begin{array}{l}525 \\
(2,4)\end{array}$ & $\begin{array}{c}522 \\
(5,2)\end{array}$ & 500 \\
\hline Mathématiques & $\begin{array}{l}517 \\
(2,7)\end{array}$ & $\begin{array}{l}533 \\
(3,5)\end{array}$ & $\begin{array}{l}533 \\
(1,4)\end{array}$ & $\begin{array}{l}537 \\
(3,1)\end{array}$ & $\begin{array}{l}529 \\
(2,5)\end{array}$ & $\begin{array}{l}510 \\
(2,5)\end{array}$ & $\begin{array}{l}536 \\
(2,1)\end{array}$ & $\begin{array}{l}493 \\
(7,6)\end{array}$ & $\begin{array}{l}547 \\
(2,8)\end{array}$ & $\begin{array}{l}557 \\
5,5)\end{array}$ & 500 \\
\hline Sciences & $\begin{array}{l}500 \\
(3,2)\end{array}$ & $\begin{array}{l}528 \\
(3,5)\end{array}$ & $\begin{array}{r}529 \\
(1,6)\end{array}$ & $\begin{array}{l}528 \\
(2,4)\end{array}$ & $\begin{array}{l}532 \\
(2,7)\end{array}$ & $\begin{array}{c}512 \\
(2,5)\end{array}$ & $\begin{array}{l}532 \\
(2,5)\end{array}$ & $\begin{array}{l}499 \\
(7,3)\end{array}$ & $\begin{array}{l}552 \\
(2,7)\end{array}$ & $\begin{array}{l}550 \\
(5,5)\end{array}$ & 500 \\
\hline
\end{tabular}

(1) Le Royaume-Uni accueille trois systèmes éducatifs légèrement différents : Angleterre et Pays de Galles, Écosse, Irlande du Nord. C'est l'ensemble du Royaume-Uni qui est pris en compte par PISA.

Source : OECD, 2001a.

Lecture : Ces « scores " ont été standardisés de telle sorte que le score moyen de l'ensemble international des élèves testés soit 500 et l'écarttype 100. La performance de l'élève finlandais médian est donc supérieure d'un demi-écart type à celui de l'élève médian de la population des 31 pays, ce qui signifie que la performance de cet élève est supérieure à celle de $70 \%$ des élèves de la population de ces pays. Comme ces performances sont obtenues sur un échantillon d'élèves, elles sont affectées d'une marge d'erreur. Les erreurs-types sont indiquées entre parenthèses. Celle de la France en Compréhension de l'écrit est de 2,7, ce qui signifie que le score "réel » moyen des élèves français a $95 \%$ de chances de se situer entre 502,3 et 507,7. Pour que la performance d'un pays soit significativement supérieure à celle de la France il faut que l'écart entre les valeurs moyennes soit supérieur à 2 fois la racine carrée de la somme des carrés des erreurs-types. Les performances significativement supérieures à celles de la France sont en caractères gras.

L'écart moyen que nous avons avec ces pays qui nous surpassent est situé entre le tiers et le quart de ces écarts. II s'agit donc d'écarts ni négligeables ni, disons, décourageants.

Une première explication à ces écarts peut être que les conditions sociales sont plus favorables dans ces pays. De même que le score moyen au bac des élèves d'un lycée doit être corrigé en fonction de leur âge et de leur origine sociale si l'on veut en déduire une information sur l'efficacité de l'enseignement dans ce lycée, de même, il peut paraître juste, si l'on veut juger du système éducatif d'un pays, de pondérer son score moyen observé, celui du tableau 1 , par des variables comme la proportion, dans ce pays, de familles monoparentales, d'immigrés récents ou, à l'inverse, d'enfants de personnes qui ont fait de longues études.

Aussi le rapport PISA indique-t-il, pour chaque pays, la performance moyenne en compréhension de l'écrit qui serait la sienne si le niveau d'un ensemble de variables sociales y était égal à la valeur internationale, compte tenu de l'effet net de chacune de ces variables dans ce pays (6).

La comparaison avec les pays ci-dessus est alors la suivante.

Cette façon de mesurer la performance moyenne n'affecte pas le score de la France elle-même. Elle

Tableau II. - Les performances corrigées en compréhension de l'écrit

\begin{tabular}{|l|c|c|c|c|c|c|c|c|c|c|}
\hline & France & Australie & Canada & $\begin{array}{c}\text { Nouvelle- } \\
\text { Zélande }\end{array}$ & $\begin{array}{c}\text { Royaume- } \\
\text { Uni }\end{array}$ & Suède & Finlande & USA & Corée & Japon \\
\hline $\begin{array}{l}\text { Performance moyenne } \\
\text { corrigée }\end{array}$ & 505 & $\mathbf{5 1 8}$ & $\mathbf{5 2 3}$ & $\mathbf{5 3 1}$ & $\mathbf{5 2 3}$ & $\mathbf{5 1 1}$ & $\mathbf{5 4 3}$ & 512 & $\mathbf{5 2 5}$ & $\mathbf{5 3 4}$ \\
\hline Erreur Type & 2,1 & 3,0 & 1,6 & 2,0 & 1,9 & 2,1 & 2,4 & 4,0 & 2,5 & 6,0 \\
\hline
\end{tabular}

Source : OECD,2001a; table 8.2.

Lecture: Les variables correctrices sont: I'index de statut socio-économique (ISEI) des parents, le nombre de leurs années d'études, le \% d'élèves appartenant à des familles monoparentales, le nombre de frères et sœurs, le \% d'élèves nés à l'étranger, l'importance des ressources éducatives (endroit tranquille pour étudier, bureau pour étudier, dictionnaire, manuels scolaires et calculatrices) à la maison, l'importance des ressources culturelles (livres de littérature, livres de poésie, œuvres d'art) à la maison. 
diminue l'écart de son score avec celui d'un certain nombre de pays (Australie, Canada, Suède), mais elle le creuse avec celui du Japon, et il s'en faut de peu que celui des États-Unis, ainsi corrigé, ne soit significativement supérieur au nôtre.

Cependant, ce type de correction est lui-même entaché d'imprécisions. D'abord, on n'est jamais sûr d'avoir pris en compte toutes les variables externes pertinentes. Ensuite, il suppose que l'effet des variables par lesquelles on corrige soit exogène. Or, ce n'est pas toujours le cas. Si par exemple un système éducatif cantonne tous ses élèves immigrés dans des écoles très inefficaces, la variable " élèves nés à l'étranger " aura dans ce système un effet négatif particulièrement prononcé, et le calcul cidessus surestimera son efficacité, parce qu'il attribuera au fait d'être étranger ce qui devrait être attribué aux écoles inefficaces que les étrangers fréquentent. De même, si la ségrégation sociale est forte dans un système éducatif et que les élèves les plus "favorisés " y choisissent les écoles les plus efficaces, le score corrigé sera très différent du score brut, pour partie indûment.

Pour cette raison, ce type de mesure n'enlève pas tout intérêt aux variables brutes. En fait, ce que nous pouvons dire de façon à peu près sûre est que la différence entre l'efficacité des systèmes éducatifs de deux pays est comprise entre celle qu'indiquent les scores bruts et celle qu'indiquent les scores corrigés. Comme on vient de le voir, la liste des pays qui nous surpassent, au moins en compréhension de l'écrit, est la même dans les deux cas.

\section{COMMENT EXPLIQUER CES ÉCARTS ?}

Les performances mesurées par PISA décrivent bien l'efficacité de l'apprentissage dans chaque pays, puisqu'il n'y a pas de raison de penser qu'il existe des différences significatives entre les pays quant aux aptitudes initiales des enfants ou à leur niveau d'intelligence générale. En revanche, l'efficacité de l'apprentissage peut être liée à des facteurs scolaires mais aussi à des facteurs sociaux.

Pour expliquer ces différences d'efficacité, la démarche canonique serait de construire un modèle multiniveau expliquant les scores des élèves par leurs caractéristiques individuelles d'une part, celle de leur classe ou de leur établissement d'autre part, et enfin celles du système éducatif de leur pays. C'est ce qui a été fait au chapitre huit de OECD, 2001a. Mais, à l'inverse des mesures canoniques de l'efficacité,
PISA dispose d'une seule observation des compétences des élèves. De ce fait, les données PISA, comme celles des évaluations internationales précédentes, ne permettent pas de repérer les caractéristiques des écoles et des classes dans lesquelles les élèves progressent le plus à niveau initial égal, même si elles peuvent atténuer ce handicap en prenant en compte d'autres prédicteurs externes comme l'origine sociale. Elles permettent seulement de repérer les caractéristiques des établissements qui scolarisent, sous contrôle de diverses variables sociales, les meilleurs élèves. Ce pourquoi, par exemple, l'analyse de OECD, 2001a, ne perçoit pas l'effet de variables dont on sait pourtant qu'elles agissent positivement sur les performances des élèves, comme la pression à la réussite ou le soutien des enseignants. De ces variables, cette analyse montre qu'elles sont plus fortes pour les élèves faibles que pour les élèves forts, mais ne peut montrer que, à niveau de départ identique, elles ont un effet positif sur les progrès des élèves. La raison en est l'effet " suppressif » qui vient précisément de ce qu'elles sont utilisées pour les élèves qui en ont le plus besoin. C'est pourquoi nous utiliserons une méthode statistiquement beaucoup plus rustique, mais, pensons-nous, plus heuristique dans ce cas précis.

Nous nous intéresserons à des variables dont des recherches empiriques précédentes ont montré qu'elles contribuaient à expliquer la performance des élèves relativement à leur niveau initial, soit, pour le dire de façon moins rigoureuse, qu'elles définissaient des conditions d'enseignement efficaces, et, de façon plus technique, que leur effet net, soit leur effet sous contrôle des autres variables dont on peut penser qu'elles agissent aussi sur l'apprentissage, était significativement positif.

Si la valeur d'une de ces variables est significativement plus favorable dans un pays donné qu'en France, on estimera qu'il y a quelques chances que ce soit une partie de l'explication de ce que les élèves de ce pays ont des compétences plus fortes que celles des élèves français.

Nous prendrons en compte, pour tenter d'expliquer les écarts de performances, des variables de quatre types :

- les caractéristiques organisationnelles du système éducatif, en particulier le caractère plus ou moins centralisé des principales décisions ;

- les attentes, la pression, l'aide reçues de la famille, ou de l'environnement social direct ; 
- l'efficacité de l'enseignement tel qu'il se déroule dans chaque classe ;

- le climat, la discipline qui prévalent en classe, qui témoignent de l'engagement des élèves.

L'influence des caractéristiques organisationnelles a été mise en évidence par Wössmann (2001) au moyen d'un modèle multiniveau appliqué aux données de la plus récente étude de l'IEA sur l'apprentissage des mathématiques et des sciences (TIMSS, 1995). Selon cette analyse, les performances des élèves sont plus fortes, aussi bien en mathématiques qu'en sciences, quand le contenu des examens et des programmes est décidé au niveau central, quand la gestion du personnel (recruter les enseignants, par exemple) est décidée au niveau de l'établissement, et quand le choix des manuels et la construction des situations d'apprentissage sont laissées à la responsabilité des enseignants. Les caractéristiques organisationnelles que nous utiliserons ici sont les suivantes :

- les examens sont-ils centralisés ? (EXAMNAT) ;

- les programmes d'enseignement sont-ils décidés au niveau national (PROGRNAT) ?

- les établissements sont-ils autonomes dans la gestion du personnel ? (PERSETAB);

- les professeurs sont-ils libres de choisir les manuels ? (MANUEENS) ;
L'influence de la stimulation et de l'aide reçues de l'environnement familial ont été mises en évidence par des études expérimentales (cf. Bloom, 1986), mais aussi par des études qui ont expliqué ainsi les écarts de performances entre certains pays : la plus grande exigence des parents à l'égard du travail scolaire de leurs enfants expliquerait une partie de la supériorité du Japon ou de la Corée sur les États-Unis (Holloway et al., 1990). Enfin, Grisay (1997) a montré que la qualité de l'environnement éducatif familial (attentes, qualité du suivi, importance accordée aux études) agissait en France positivement sur les apprentissages des collégiens en maths et en français. Les caractéristiques de l'environnement familial que nous utiliserons ici sont :

- les parents ont-ils des discussions fréquentes avec leurs enfants sur des questions culturelles?

- intensité du soutien scolaire reçu de sa famille par l'élève ;

- temps consacré au travail à la maison ;

- richesse des ressources éducatives disponibles à la maison.

L'influence des deux catégories suivantes de variables a été établie par les recherches sur l'efficacité des établissements primaires et secondaires (cf. une synthèse internationale, Bosker et Scheerens, 1997, et pour les collèges français, Grisay, 1997). En utilisant des modèles multiniveaux qui permettent de

\section{Tableau III. - Correspondance entre les variables PISA sur l'efficacité de l'enseignement et la discipline des élèves et les variables correspondantes associées à l'efficacité dans les collèges français au début des années 1990 (1)}

\begin{tabular}{|l|l|}
\hline \multicolumn{1}{|c|}{ Variables PISA } & \multicolumn{1}{|c|}{ Grisay, 1997} \\
\hline \multicolumn{1}{|c|}{\begin{tabular}{l} 
Efficacité de l'enseignement en classe \\
\hline Pression pour la réussite
\end{tabular}} & $\begin{array}{l}\text { En cours, on travaille sans arrêt ; en cours, il faut se fatiguer pour réussir ; si je } \\
\text { remettais un travail mal fait, je sais que les professeurs ne l'accepteraient pas. }\end{array}$ \\
\hline Soutien par les enseignants & $\begin{array}{l}\text { Les professeurs sont attachés à la réussite de tous les élèves; on peut leur } \\
\text { parler après le cours; ils se tracassent si les élèves ont des mauvais résultats; } \\
\text { ils nous aident à faire de notre mieux; ils ont à cœur de nous aider dans notre } \\
\text { travail. }\end{array}$ \\
\hline $\begin{array}{l}\text { Qualité des relations } \\
\text { élèves/enseignants }\end{array}$ & $\begin{array}{l}\text { Les élèves ont le sentiment d'être traités avec justice et considération; mes } \\
\text { professeurs m'écoutent. }\end{array}$ \\
\hline $\begin{array}{l}\text { Qualité de la discipline } \\
\text { et engagement des élèves }\end{array}$ & $\begin{array}{l}\text { Pas de perte de temps en classe ; les professeurs se font respecter; le cours } \\
\text { commence immédiatement ; les élèves font attention à ce que dit le professeur. }\end{array}$ \\
\hline
\end{tabular}

(1) La parenté des composites apparaîtra mieux en se référant au détail des composites PISA qui est donné dans le tableau en annexe. 
traiter de façon appropriée les effets de ce qui se passe dans les classes et de ce qui relève du niveau établissement lui-même, ces recherches vont au-delà de l'étude de "l'effet établissement » et permettent de distinguer les facteurs qui jouent aux niveaux de l'élève, de la classe (donc aussi de l'enseignant) et de l'établissement.

Le tableau III met en évidence la correspondance entre les variables de ces deux catégories que nous utiliserons pour la comparaison et les variables associées à l'efficacité des collèges français selon Grisay (1997), correspondance évidemment essentielle pour l'approche retenue ici.

\section{UNE COMPARAISON AVEC LES HUIT PAYS QUI SURPASSENT LA FRANCE ET AVEC LES USA}

Le tableau IV présente une comparaison de ces variables favorables à la réussite des élèves entre la France et chacun des pays considérés dans cette étude (7).

La configuration organisationnelle de l'enseignement semble plus favorable au Royaume-Uni et en Nouvelle Zélande qu'en France, mais, pour les autres pays, ce n'est pas le cas. II est clair, par exemple, selon cette analyse, que l'Australie et le Canada devraient nous envier le baccalauréat et l'existence de programmes nationaux.

II semble par ailleurs que les jeunes Français bénéficient d'un environnement familial en général plus favorable que celui que reçoivent les élèves de ces pays : les parents et leurs enfants ont plus fréquemment des discussions sur des films ou des livres, sur des évènements politiques et sociaux; les élèves disposent plus fréquemment chez eux de dictionnaires, de calculatrices, d'un bureau, d'un endroit calme pour étudier ; dans un seul pays (le RoyaumeUni) le temps de travail à la maison est plus long; enfin, il semble que nous occupions une position médiane quant à l'aide que les enfants disent trouver auprès de leurs parents. Ce soutien est cependant plus fort au Royaume-Uni, en Suède, en Australie et en Nouvelle-Zélande qu'en France et cela contribue sans doute à expliquer le meilleur score de ces pays.

Les enseignants efficaces aident leurs élèves à réussir, ils ont de bonnes relations avec eux, en même temps qu'ils sont exigeants. C'est le portrait qu'en tracent deux recherches françaises: celle de Felouzis (1996) sur les classes de seconde et celle de Grisay (1997) sur les collèges. Ces trois qualificatifs correspondent aux trois construits de notre tableau. Force est de constater que ce type d'enseignants, loin d'être absent en France, y est cependant moins fréquent que dans les pays du Commonwealth et dans les pays scandinaves de notre panel, du moins d'après les déclarations des élèves. Mais on peut penser que la norme des élèves est indexée sur la culture des enseignants et donc que, si écart il y a avec la "réalité " par suite d'un effet de contexte national, l'écart réel entre deux pays est minimisé par les réponses des élèves et non exagéré. En bref, ceci suggère que les enseignants qui se préoccupent davantage d'enseigner leur discipline que de la faire apprendre par leurs élèves, pour qui l'exigence porte plus sur le niveau du cours délivré que sur le travail attendu des élèves, sont plus nombreux en France que dans ces pays.

Les élèves sont plus nombreux à estimer que la discipline est satisfaisante (le fait que l'on peut écouter le professeur, qu'on peut commencer le cours à l'heure, qu'il n'y a pas de bruit et de désordre en classe, ....) en France qu'au Canada, en Nouvelle Zélande, en Suède et en Finlande. En revanche, les jeunes Coréens et Japonais, comme les jeunes Britanniques, sont plus nombreux que les jeunes Français à trouver la discipline propice au travail scolaire. Les chefs d'établissements français se plaignent moins souvent que leurs homologues des pays auxquels nous comparons ici la France que les élèves manquent de respect à leurs professeurs, qu'ils perturbent les cours, qu'ils boivent ou fument des substances illégales, qu'ils sautent des cours, qu'ils sont absents, qu'ils martyrisent d'autres élèves, à l'exception des chefs d'établissements des deux pays asiatiques, selon lesquels ces phénomènes sont peu présents dans leur pays. Si donc une meilleure discipline des élèves semble pouvoir contribuer à expliquer la meilleure performance des jeunes Coréens et Japonais, il ne semble pas qu'on puisse invoquer cette raison pour les pays du Commonwealth et les pays scandinaves : au contraire, les élèves paraissent y avoir plus fréquemment qu'en France un comportement défavorable à l'apprentissage.

Bref, ce que suggère notre comparaison, c'est que par rapport aux pays du Commonwealth et aux pays scandinaves efficaces (8), la moindre réussite des élèves français s'explique sans doute moins, parmi les variables que nous avons examinées, par l'organisation du système éducatif, ou le comportement des élèves, ou encore le désintérêt des parents, que par ceci que les enseignants exigent moins de travail de leurs élèves, en même temps 
Tableau IV. - Variables favorables à la réussite des élèves.

\section{Comparaison de la France avec les pays dont les élèves ont en moyenne des compétences plus élevées}

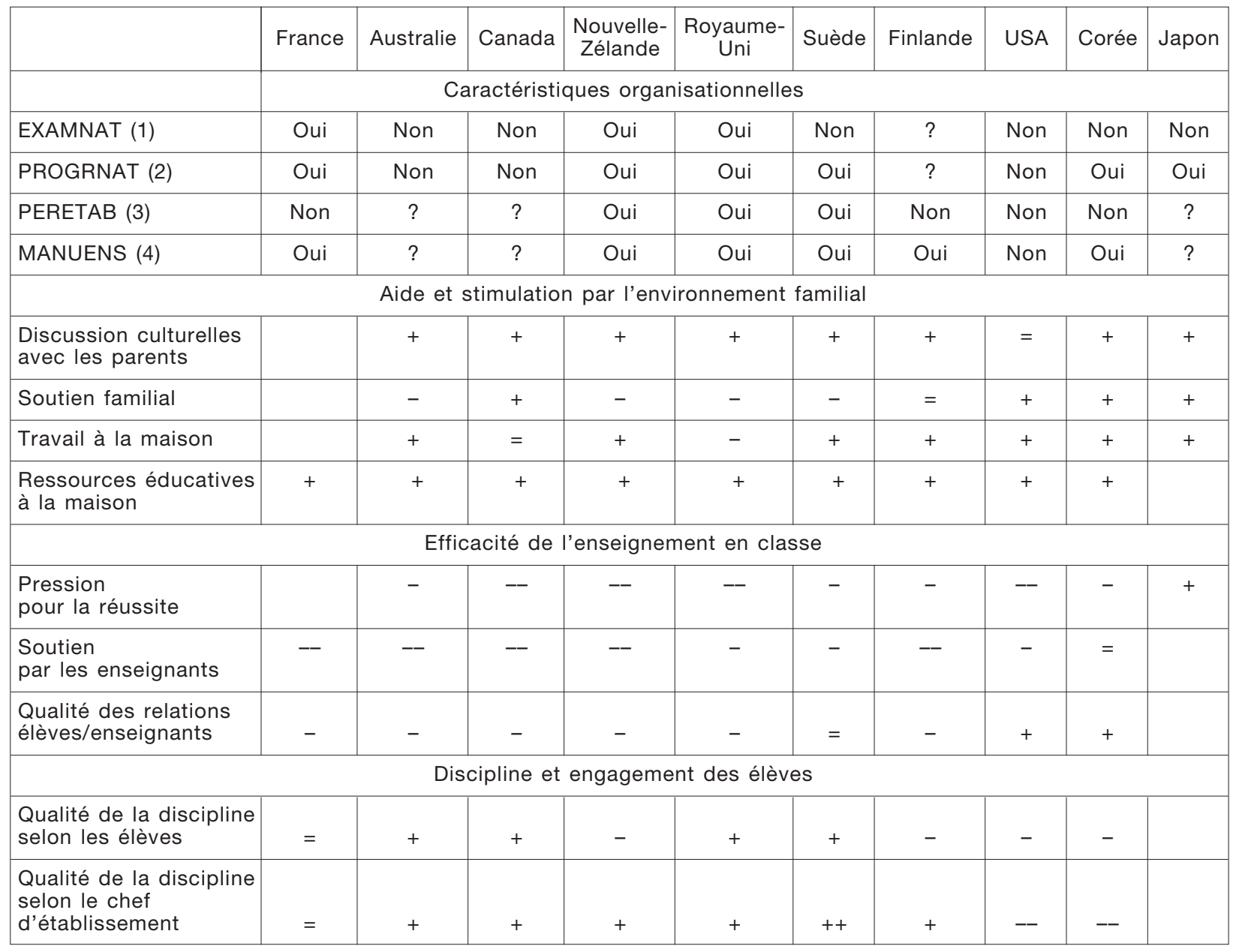

(1) Source : TIMSS report, p 19, situation en 1994-1995.

(2) Source: TIMSS report, p 17, situation en 1994-1995.

(3) Source : OCDE-CERI, Regards sur l'éducation, 1998.

(4) Source : OCDE-CERI, Regards sur l'éducation, 1998. La réponse ne permet pas de distinguer les cas où la décision est prise individuellement par chaque enseignant ou collectivement par les enseignants d'un établissement.

Source : Calculs à partir de la base de données PISA, sauf mention contraire.

Lecture: oui ou non indiquent si, dans le pays, les décisions considérées sont prises ou non au niveau le plus favorable à l'efficacité de l'enseignement. ? signifie que les données ne sont pas disponibles. Dans les parties inférieures du tableau, les + et les - se lisent ainsi : + signifie que les réponses en France sont significativement plus favorables à l'efficacité de l'enseignement que dans ce pays ; ++ ; idem, mais l'écart est supérieur à 0,5 écart-type ; - signifie : les réponses en France sont significativement moins favorables à l'efficacité de l'enseignement que dans ce pays ; - : idem, mais l'écart est supérieur à 0,5 écart-type.

Pour aider à la lecture, on n'a pas fait figurer les valeurs des indices dans le tableau. Ces indices composites sont calculés à partir des réponses aux questions indiquées en annexe. Leur définition procède de considérations théoriques et de recherches empiriques précédentes, qui en avaient montré la pertinence. Le fait qu'ils "faisaient sens ", c'est-à-dire que l'ensemble des réponses aux questions d'un indice construisaient une valeur latente valide et dont le comportement était conforme à ce qui était attendu, a été vérifié en utilisant un modèle d'équations structurelles (dont un exemple souvent utilisé en France est LISREL). Leur valeur a été calculée en utilisant le modèle de Rash et l'estimation de Warm, cette dernière choisie en particulier pour son bon comportement aux extrémités de l'échelle. Ces valeurs ont été standardisées de façon à présenter une moyenne internationale de 0 et un écart-type de 1 . Pour les "discussions culturelles avec les parents ", par exemple, l'indice composite vaut 0,27 pour la France (avec une erreur-type de 0,019) et 0,06 pour le Royaume-Uni (avec une erreur-type de 0,017). Comme la différence $0,27-0,06=0,21$ est supérieure à deux fois la racine carrée de $\left(0,019^{2}+0,017^{2}\right)$, cet indice est significativement plus favorable en France qu'au Royaume-Uni à l'efficacité de l'enseignement, ce que signifie le + dans la colonne Royaume-Uni. Les questions qui construisent chaque variable sont indiquées en annexe 3. 
qu'ils les aident moins à progresser, qu'ils se soucient moins de leur bien-être, les écoutent moins, les traitent moins équitablement - ou plutôt que, en France, moins nombreux sont les élèves à trouver que leurs enseignants se comportent ainsi.

Ces résultats doivent cependant être mis en perspective à partir des considérations qui suivent.

D'une part, notre modèle n'inclut pas toutes les variables qui agissent sur les performances des élèves. L'une d'elles est le temps d'enseignement (cf. Delhaxe, 1997, pour une revue des recherches sur cette variable) que reçoivent les élèves. Une autre, à l'effet encore plus direct ici, est le redoublement. Dans l'échantillon français, $55 \%$ des élèves testés sont en seconde ou en première, $37 \%$ sont en troisième et $7 \%$ en quatrième. Selon les études empiriques sur les effets du redoublement, celui-ci a pour principal effet de faire perdre une année aux élèves sans bénéfice en termes de performance (cf. Crahay, 1996, pour une revue de la littérature à ce sujet) : un élève qui redouble le CP arrive au CM2 avec le même niveau qu'un élève de même niveau scolaire qu'on a laissé passer en CE1, mais, évidemment, un an plus tard. Si l'on étend ces résultats, il est vrai observés surtout en primaire, la pratique du redoublement au collège expliquerait à elle seule une bonne partie de notre retard sur ces pays. D'autant qu'on peut aussi avancer l'hypothèse que le fait que le redoublement n'existe pas, ou quasiment pas, dans les pays scandinaves ou du Commonwealth, encourage les enseignants au type d'attitudes et de pratiques que notre modèle associe à l'efficacité : il faut davantage s'efforcer de tirer chacun vers le haut lorsqu'aucun dispositif institutionnel n'est prévu pour la relégation des élèves en difficulté.

Autrement dit, il nous semble possible d'affirmer que, parmi les variables de notre modèle, celles qui décrivent l'attitude des enseignants vis-à-vis des élèves sont celles qui expliquent le mieux l'écart avec ces pays, mais, d'une part, elles peuvent dépendre d'autres variables sur lesquelles il serait plus opportun d'agir, d'autre part des variables qui ne figurent pas dans le modèle peuvent aussi contribuer à expliquer les écarts.

II y a une autre limite à cette étude. Notre raisonnement repose sur l'idée que les variables sur le fonctionnement du système éducatif recueillies auprès des élèves de 15 ans décrivent un état, une culture, des comportements, valables pour l'ensemble du système éducatif de leur pays, ou au moins pour l'ensemble de l'enseignement secon- daire, et pas seulement pour les types d'établissements où sont scolarisés les élèves de 15 ans.

II s'agit là d'une hypothèse et, au surplus, d'une hypothèse plus ou moins plausible selon les pays. Par exemple, elle a plus de chances d'être vraie en Suède, où les élèves de 15 ans sont en dernière année de la «Grundskola » qu'ils fréquentent depuis l'âge de 7 ans, qu'en France, où, si une petite moitié des élèves testés est encore au collège, une grosse moitié est en seconde, dans une culture de lycée ou de lycée professionnel qu'ils ne fréquentent que depuis le début de l'année (9), et dont la culture et les pratiques peuvent être différentes de celles du collège.

Cependant, il nous semble que, tout en impliquant une certaine prudence dans l'analyse des résultats, cette hypothèse peut être faite. Nous savons d'une part que la culture des enseignants français diffère fortement de celle des enseignants de pays de culture anglaise (cf. Broadfoot et Osborne, 1993, pour une comparaison des instituteurs anglais et français, par exemple (10)), d'autre part qu'il y a une assez forte homogénéité dans les attitudes, les valeurs, les pratiques des enseignants français à tous les niveaux du secondaire (Dubet et Martuccelli, 1996, par exemple). Nous pouvons donc porter, nous semble-t-il, un jugement sur l'enseignement secondaire français à partir de ces analyses.

\section{EFFICACITÉ ET ÉQUITÉ}

Si les élèves français ont en moyenne des compétences inférieures à celles des élèves de ces pays, est-ce parce que notre système éducatif sacrifie le plus grand nombre sur l'autel de l'élitisme? La réponse est négative - ou alors cet élitisme serait très inefficace - puisque nos meilleurs élèves ont aussi des compétences moindres que celles des leurs.

On peut voir dans le tableau $\mathrm{V}$ que, à quelques exceptions près - Japon et Corée pour la compréhension de l'écrit, Suède pour les sciences -, les performances des $10 \%$ les meilleurs parmi les élèves sont significativement inférieures en France à ce qu'elles sont dans les pays où les compétences moyennes des élèves sont supérieures à celles des nôtres.

En compréhension de l'écrit, l'écart des performances de ces élèves entre la France et la plupart de ces pays est supérieur à l'écart qui existe entre les performances moyennes indiquées dans le tableau I. Ce n'est pas le cas en Suède, en Corée et au Japon, où la distribution des compétences est moins inéga- 
Tableau V. - Les performances de l'élite scolaire

( $90^{\mathrm{e}}$ décile de la distribution des performances)

\begin{tabular}{|l|c|c|c|c|c|c|c|c|c|c|c|}
\hline & France & Australie & Canada & $\begin{array}{c}\text { Nouvelle- } \\
\text { Zélande }\end{array}$ & Uni (1) & Suède & Finlande & USA & Corée & Japon & $\begin{array}{c}\text { Moyenne } \\
\text { internationale }\end{array}$ \\
\hline $\begin{array}{l}\text { Compréhension } \\
\text { de l'écrit }\end{array}$ & 619 & 656 & 652 & 661 & 651 & 630 & 654 & 636 & 608 & 625 & 622 \\
\hline Mathématiques & 629 & 647 & 640 & 659 & 646 & 626 & 637 & 620 & 650 & 662 & 628 \\
\hline Sciences & 631 & 646 & 641 & 653 & 656 & 630 & 645 & 628 & 652 & 659 & 631 \\
\hline
\end{tabular}

Source : OECD, 2002a, tables 3.3,3.1, 2.3a.

Lecture: Mêmes indications que pour le tableau 1.

litaire que dans ces autres pays. En revanche, les meilleurs élèves des États-Unis, où cette distribution est très inégalitaire (tableau $\mathrm{VI}$ ), font mieux que les meilleurs élèves français.

En poursuivant la comparaison entre les tableaux I et $V$, on voit que, en mathématiques, l'écart entre les "groupes de tête " (tableau V) est du même ordre de grandeur que l'écart entre les performances moyennes (tableau I) - à l'exception du Japon et de la Corée, où il est un peu plus faible. On voit aussi que, en sciences, l'écart entre les groupes de tête est en général plus faible que l'écart entre les performances moyennes.

Si donc nous ne semblons pas avoir sacrifié les performances moyennes sur l'autel de l'élitisme, estce parce qu'au contraire notre système éducatif les aurait sacrifiées sur l'autel de l'équité ? En fait, cette question est mal posée. L'étude des résultats de TIMSS (Vandenberghe et Zacchary, 2001), comme de ceux de PISA (OECD, 2002a) montre qu'il n'y a pas vraiment à " choisir " entre efficacité (moyenne) et équité, dans la mesure où un nombre significatif de pays réussissent (ou, à l'inverse, échouent) dans ces deux dimensions à la fois. II vaut donc mieux se demander si nous compensons, en quelque sorte, notre efficacité inférieure à celle de ces pays par une équité qui serait supérieure à la leur.

La question se pose d'autant plus que la France manifeste depuis vingt ans un fort souci des élèves faibles ou des élèves de zone difficile, que les pays scandinaves ont une forte réputation d'équité (non ségrégation, égalité des chances...) alors que les pays du Commonwealth, mais aussi la Suède, se sont lancés depuis une quinzaine d'années dans des politiques dites en France "libérales ", que I'on soupçonne de creuser les inégalités entre élèves.
Selon un schéma proposé dans Meuret (2001), nous appréhenderons l'équité de ces systèmes éducatifs en considérant que la situation est plus équitable dans un système que dans un autre si trois types d'inégalités y sont plus faibles: l'écart de compétences entre les meilleurs et les moins bons élèves, d'autant plus pertinent ici que PISA mesure ces compétences vers la fin de la scolarité obligatoire ; la proportion d'élèves situés sous un seuil de compétences que l'on peut juger minimum pour mener une vie digne dans la société ; la dépendance entre le niveau de compétences et l'environnement familial et social de l'élève.

Des mesures de ces inégalités sont proposées dans le tableau VI.

La comparaison en matière de dispersion situe différemment la France selon les domaines. En maths et en compréhension de l'écrit, la dispersion est plutôt plus faible que dans les pays du Commonwealth et qu'aux USA, se compare aux dispersions scandinaves et est plus forte que dans les pays asiatiques. En sciences, en revanche, la dispersion est plutôt plus forte que dans les pays du Commonwealth, aussi forte qu'aux États-Unis, plus forte que dans les pays scandinaves et asiatiques. Pour qui accorderait le même poids à chacun de ces trois domaines, l'écart entre les faibles et les forts serait donc, en France, plutôt moindre que dans les pays du Commonwealth et qu'aux USA, plutôt plus fort que dans nos deux pays scandinaves et que dans nos deux pays asiatiques.

La comparaison en ce qui concerne la proportion d'élèves très faibles en maths est favorable par rapport aux États-Unis, et nous situe au même rang que les pays du Commonwealth; seuls la Finlande et le Japon font nettement mieux que nous en la matière. Si l'on prend pour critère la proportion d'élèves dont la per- 
Tableau VI. - Inégalités des compétences entre élèves dans divers pays

\begin{tabular}{|c|c|c|c|c|c|c|c|c|c|c|}
\hline & France & Australie & Canada & $\begin{array}{l}\text { Nouvelle- } \\
\text { Zélande }\end{array}$ & $\begin{array}{c}\text { Royaume- } \\
\text { Uni }\end{array}$ & Suède & Finlande & USA & Corée & Japon \\
\hline \multicolumn{11}{|c|}{ Dispersion individuelle (écart-type des scores individuels, (erreur-type)) } \\
\hline Mathématiques & $\begin{array}{c}89 \\
(1,9)\end{array}$ & $\begin{array}{c}90 \\
(1,6)\end{array}$ & $\begin{array}{c}85 \\
(1,1)\end{array}$ & $\begin{array}{c}99 \\
(1,9)\end{array}$ & $\begin{array}{c}92 \\
(1,6)\end{array}$ & $\begin{array}{c}93 \\
(2,5)\end{array}$ & $\begin{array}{c}80 \\
(1,4)\end{array}$ & $\begin{array}{c}98 \\
(2,4)\end{array}$ & $\begin{array}{c}84 \\
(2,0)\end{array}$ & $\begin{array}{c}87 \\
(3,1)\end{array}$ \\
\hline Sciences & $\begin{array}{c}102 \\
(2,0)\end{array}$ & $\begin{array}{c}94 \\
(1,6)\end{array}$ & $\begin{array}{c}89 \\
(1,1)\end{array}$ & $\begin{array}{c}101 \\
(2,3)\end{array}$ & $\begin{array}{c}98 \\
(2,0)\end{array}$ & $\begin{array}{c}93 \\
(1,4)\end{array}$ & $\begin{array}{c}86 \\
(1,2)\end{array}$ & $\begin{array}{c}101 \\
(2,9)\end{array}$ & $\begin{array}{c}81 \\
(2,7)\end{array}$ & $\begin{array}{c}90 \\
(3,0)\end{array}$ \\
\hline \multicolumn{11}{|c|}{ Compétences des élèves les plus faibles ( $\%$ d'élèves dont le score est...) } \\
\hline$<400$ points en maths & $10 \%$ & 16 & 6 & 7 & 9 & 12 & 5 & 18 & 8 & 5 \\
\hline \multicolumn{11}{|c|}{$\begin{array}{l}\text { Part de variance des performances en compréhension de l'écrit } \\
\text { expliquée par l'environnement familial et social }\end{array}$} \\
\hline ISEI (2) & $13 \%$ & 10 & 7 & 10 & 15 & 9 & 6 & 11 & 4 & 1 \\
\hline IESCS (3) & $22 \%$ & 17 & 11 & 16 & 19 & 11 & 9 & 22 & 9 & 6 \\
\hline \multicolumn{11}{|c|}{$\begin{array}{l}\text { Différences de performances en compréhension de l'écrit } \\
\text { pour une unité de l'indice social ESCS }\end{array}$} \\
\hline IESCS & 47 & 46 & 37 & 45 & 49 & 36 & 30 & 48 & 21 & 21 \\
\hline
\end{tabular}

(1) En compréhension de l'écrit seulement, PISA complète l'approche relative des niveaux de compétences par une approche normative, pour laquelle 5 niveaux ont été définis, dont le niveau 1 est le plus faible : les élèves qui ont atteint, mais non dépassé ce niveau sont capables " de repérer des informations simples, d'identifier le thème principal d'un texte ou d'établir un lien simple avec une connaissance de tous les jours " (OECD, 2002a, p. 48) mais non de compétences plus élevées.

(2) ISEI : Cet indice, utilisé pour les études internationales sur la mobilité sociale, mesure sur une échelle continue la catégorie socioprofessionnelle des parents. II prend en compte celle du parent qui a la plus haute.

(3) IESCS : Index of economic, social and cultural status. Cet indice est composé à partir de la position sociale des parents (ISEI), du niveau d'éducation du parent qui a la plus élevée, du niveau de vie de la famille (mesuré à partir d'objets possédés), du montant des ressources liées à l'éducation à la maison, du montant des ressources liées à la culture à la maison. Le calcul porte sur la performance en compréhension de l'écrit. Source: OECD, 2002a. tables 2.3a,3.1,3.3,5.2b, 8.1 et calculs spécifiques ; fig.2.3.

Lecture : L'écart-type de la distribution des scores en Compréhension de l'écrit est de 92 points en France, donc légèrement inférieur à l'écarttype de 100 qui est, par convention d'échelle, celui de la distribution internationale. Les scores australiens sont significativement plus dispersés, puisque l'écart 102-92 = 10 est supérieur à deux fois la racine carrée de la somme des carrés des erreurs-types $(1,7$ et 1,6$) ;$ c'est pourquoi ils sont en caractères gras. $10 \%$ des élèves français ont un score inférieur à 400 sur l'échelle de moyenne 500 utilisée dans les tableaux 1 et 2. $15 \%$ des élèves français ne dépassent pas le premier niveau de compréhension de l'écrit, le plus faible d'une échelle qui en compte 5 . Enfin, l'environnement familial explique en France $22 \%$ de la variance de la performance des élèves en compréhension de l'écrit : les performances en ce domaine y dépendent donc davantage du milieu familial qu'au Canada où il n'en explique que $11 \%$. Une autre façon de mesurer l'influence de l'environnement social des élèves est de calculer de combien le score d'un élève augmente quand on augmente d'une grandeur donnée l'indice ESCS, soit, de façon imagée, quand ses parents sont un barreau plus haut dans l'échelle sociale. Cette grandeur en France (47) n'est pas significativement différente de ce qu'elle est aux USA (48), puisque, sur cette échelle, une différence doit atteindre environ cinq points pour être significative.

formance en compréhension de l'écrit est inférieure ou égale au niveau 1 , de nouveau nous faisons mieux que les USA, de nouveau la Finlande, le Japon font mieux que nous, mais la Corée, l'Australie et le Canada font aussi mieux que nous. II n'y a donc que par rapport aux États-Unis que nous faisons mieux de façon signi- ficative dans les deux domaines, quant à la capacité d'assurer à tous des compétences minimales.

L'effet de l'environnement familial et social, quel que soit l'indice par lequel on le mesure, sur les performances en lecture est aussi fort en France qu'aux 
États-Unis et que dans les pays du Commonwealth, à l'exception du Canada, plus équitable que nous à cet égard de même que le Japon, la Corée et les deux pays scandinaves. Cela est vrai dans l'approche "IESCS » où l'on s'efforce de prendre en compte tous les facteurs externes à l'école qui peuvent influencer la performance scolaire, comme dans l'approche "ISEI » où l'on s'intéresse seulement à l'effet de la position sociale des parents. Si cette situation est confirmée par les prochaines éditions de PISA, cela signifiera que l'inégalité des chances est aussi forte dans notre système éducatif que dans les pays dont nous pensons que le caractère décentralisé (les États-Unis) ou " libéral » (Nouvelle-Zélande, Royaume-Uni) les rendent inégalitaires. Les comparaisons permises par TIMSS avec les USA, le Royaume-Uni, la Nouvelle-Zélande, l'Australie étaient plus favorables (Vandenberghe et Zacchary, 2001) : l'influence de l'origine sociale était en France plus faible ou égale; dans PISA (sur une autre discipline, avec une autre façon de mesurer cette influence comme de définir la position sociale) elle est égale ou plus forte. II convient donc d'être prudent dans le diagnostic, encore que la qualité des mesures, tant de l'origine sociale que des compétences des élèves, soit sans doute supérieure dans PISA que dans TIMSS.

Sur trois dimensions de l'équité, il semble donc que dans une seule - l'écart entre les élèves les plus faibles et les plus forts -, nous puissions trouver satisfaisante cette comparaison. Du point de vue de l'équité, la situation de la France vis-à-vis de ces pays est moins systématiquement défavorable que de celui de l'efficacité, mais certains pays (le Canada, la Finlande, la Corée et le Japon) nous surpassent dans les deux dimensions.

\section{CONCLUSION}

Dans deux recherches empiriques relevant de problématiques différentes, Grisay (1997) et Felouzis (1997) ont dressé deux portraits relativement sem- blables de l'enseignant efficace (cf. Meuret, 2000) dans l'enseignement secondaire français : quelqu'un qui se préoccupe de faire apprendre tous ses élèves, de les faire progresser, plutôt que du « niveau " du cours qu'il professe ; quelqu'un de compétent, d'exigeant, dont le cours est structuré et qui obtient une forte attention de ses élèves, tout en accueillant leurs questions, en leur portant considération, attention et non mépris et distance. Ce que les données PISA ajoutent à cela est que ce type d'enseignant est, auprès des élèves de 15 ans, moins fréquent en France que dans les pays du Commonwealth, en Suède et en Finlande, alors même que, quant à d'autres facteurs de l'efficacité de l'apprentissage l'organisation du système éducatif, le soutien des parents, la discipline des élèves - notre situation est plutôt meilleure que la leur. II est donc probable que l'attitude des enseignants français vis-à-vis de l'apprentissage et vis-à-vis de leurs élèves explique en partie le niveau plus faible de leurs élèves par rapport à ceux des pays que nous venons d'évoquer. Par ailleurs, l'équité du système éducatif français n'est pas telle qu'on puisse prétendre qu'elle compense la faiblesse de l'efficacité. Certes, l'attitude des enseignants n'est pas, loin s'en faut, le seul facteur qui commande l'apprentissage ; certains pays où cette attitude est plus favorable à l'efficacité qu'en France ne sont pourtant pas plus efficaces qu'elle - c'est le cas des États-Unis - et certains pays où cette attitude n'est pas plus favorable qu'en France sont pourtant plus efficaces - c'est le cas de la Corée et du Japon. Reste que, si nous cherchons dans les comparaisons internationales des indications sur les voies à suivre pour améliorer notre système éducatif, celle-ci est une de celles qui nous sont le plus clairement indiquées. II semble que certaines des orientations actuellement prônées - renforcer l'autorité des enseignants en renforçant les sanctions, traiter la faiblesse scolaire des collégiens par l'orientation dans des filières spécifiques prennent cette voie à contre-sens.

Denis Meuret Université de Bourgogne IREDU-CNRS 
(1) On trouvera dans la brochure "PISA sample tasks ", disponible à l'OCDE ou sur www.pisa.oecd.org. une présentation des exercices et de leur correspondance avec les différents éléments de cette définition.

(2) Les compétences mesurées en sciences mobilisent des concepts empruntés à la physique, à la chimie, à la biologie et aux sciences de la terre.

(3) "Cette modernisation à l'américaine vaudra force applaudissements à ses promoteurs mais aussi, pour les autres, une production record d'analphabètes par les collèges et les lycées comme aux États-Unis » (Debray, R., A monsieur le ministre de l'éducation, Le Monde, 3 mars 1998).

(4) Pour comparer les compétences de pays dont les programmes scolaires ne sont pas exactement les mêmes, deux possibilités existent, soit comparer la maîtrise de la partie de programme commune à tous les pays - c'était la solution retenue par l'IEA -, soit évaluer des compétences générales mobilisant des concepts ou mécanismes majeurs enseignés dans tous les pays - c'est la solution retenue dans PISA.

(5) Ces comparaisons sont empruntées à OECD, 2001a, p. 57.

(6) Pour illustrer ceci d'un exemple: comme la proportion des adultes qui ont fait des études supérieures est plus élevée au Royaume-Uni qu'en France, et que le niveau d'éducation des parents est, dans tous les pays, positivement associé à la performance des enfants, il est possible que la meilleure performance du Royaume-Uni s'explique en partie par cela, et non par une meilleure qualité de son système éducatif. II convient donc, pour évaluer les systèmes éducatifs, de comparer, non pas les scores bruts du Royaume-Uni et de la France, comme nous l'avons fait dans le tableau I, mais des scores " corrigés ». Pour cela, on cal- cule le score qui serait celui de la France (du RoyaumeUni) si les parents des élèves y avaient bénéficié de la durée internationale moyenne de scolarité, compte tenu de l'effet en France (au Royaume-Uni) de la durée de la scolarité des parents sur la performance des enfants.

(7) Les données que nous utiliserons ici sont issues du questionnaire que les élèves de PISA ont rempli, sauf la description des caractéristiques organisationnelles du système que nous empruntons à OCDE-CERI, Regards sur l'éducation (1998) ou au rapport sur l'étude IEA-TIMSS.

(8) La performance des élèves coréens et japonais est plus difficile à expliquer par les variables mobilisées ici, mise à part leur plus grande discipline. S'y rajoute le fait qu'ils recoivent, comme on sait et comme PISA le confirme, de nombreuses heures de cours hors de l'école. Cependant, les élèves d'autres pays, comme la Grèce, reçoivent aussi beaucoup de cours externes sans en tirer autant de bénéfices. Quoi qu'il en soit, les variables sur l'efficacité de l'enseignement en classe ne sont pas plus favorables au Japon qu'en France et, en Corée, seulement deux d'entre elles sur les trois sont plus favorables qu'en France. Dans les deux pays, selon les élèves, la qualité des relations entre élèves et enseignants est inférieure à ce qu'elle est en France.

(9) La prise d'information a eu lieu au cours du second trimestre de l'année scolaire.

(10) Exemple : $65 \%$ des instituteurs français citent les compétences de base (lire, écrire, compter) parmi les buts de leur enseignement, contre $29 \%$ seulement pour les anglais (p. 89).

\section{BIBLIOGRAPHIE}

BLOOM B. (1986). - Le défi des deux sigmas. In M. Crahay et D. Lafontaine (eds), L'art et la science de l'enseignement. Liège: Labor.

BROADFOOT P., OSBORN M. (1993). - Perceptions of teaching, Primary school teachers in England and in France. London/NewYork: Cassell, 151 p.

CRAHAY M. (1996). - Peut-on lutter contre l'échec scolaire ? Bruxelles: De Boeck, $322 \mathrm{p}$.

DELHAXE A. (1997). - Le temps comme unité d'analyse dans la recherche sur l'enseignement. Revue Française de pédagogie, $n^{\circ} 118, p .107-125$.

DUBET F. et MARTUCCELLI D. (1996). - À l'école, Sociologie de l'expérience scolaire. Paris : Seuil.

DURU-BELLAT M. et MEURET D. (2001). - Nouvelles formes de régulation dans les systèmes éducatifs étrangers: autonomie et choix des établissements scolaires. Revue Française de Pédagogie, $n^{\circ} 135$, p. 173-223.

FELOUZIS G. (1997). - L'efficacité des enseignants. Paris: PUF, $189 \mathrm{p}$.

GRISAY A. (1990). - Des indicateurs d'efficacité pour les établissements. Éducation et Formations, $\mathrm{n}^{\circ} 22$, p. 31-46 (MEN-DEP).
GRISAY A. (1997). - Évolution des acquis cognitifs des élèves au cours des années de collège. Paris : MENDEP (Dossiers Éducation et Formations, $n^{\circ} 88$ ), 360 p.

HANUSHE E.A. et LUQUE J.A. (2002). - Efficiency and Equity in schools around the world, Working paper 8949, NBER, Cambridge, Ma., 26 p.

HOLLOWAY S.D., FULLER, B. HESS R.D., AZUMA H., KASHIWAGI, K., et GORMAN, K. (1990). - The family's influence on achievement in Japan and the United States. Comparative education review, 34 (2), p. 196208.

MEURET D. (2002). - Tentative de comparaison de l'équité des systèmes éducatifs français et américain. Carrefours de l'éducation, $\mathrm{n}^{\circ} 13$, p. 127-151 (Université d'Amiens).

MEURET D. (2001). - System of equity indicators for educational systems, p. 133-164. In N. Bottani, D. Cochran et W. Hutmacher (eds.), In pursuit of Equity in Education. Dordrecht : Kluwer publishers, 388 p.

MEURET D. (2000). - Etablissements scolaires : ce qui fait la différence. L'Année Sociologique, 50 (2), p. 545556.

OCDE-CERI (1998). - Regards sur l'éducation. Paris : OCDE-CERI, $427 \mathrm{p}$. 
OECD (2001a). - Knowledge and skills for life, First results from PISA 2000, Paris: OECD, 322 p.

OECD (2001b). - Choice of assessment tasks and the relative standing of countries in PISA 2000, a first analysis. Document pour la treizième réunion du conseil des pays participants, Paris, $10 \mathrm{p}$.

SCHEERENS J. et BOSKER R. (1997). - The foundations of educational effectiveness. Oxford : Pergamon, $347 \mathrm{p}$.
VANDENBERGH V. et ZACCHARY M.D. (2001). - Is there an Equity/Efficiency trade-off ? In N. Bottani, D. Cochran et W. Hutmacher (eds.), In pursuit of Equity in Education, p. 243-252, Dordrecht: Kluwer publishers, $388 \mathrm{p}$.

WÖSSMANN L. (2000). - Schooling Ressources, Educational Institutions and student performance : the international evidence. Kiel Working paper $n^{\circ}$ 983, Kiel Institute for World Economics, $86 \mathrm{p}$.

ANNEXe 1

\section{Qu'est-ce que PISA?}

1. Dans 32 pays, un échantillon d'élèves de 15 ans évalués en 2000 dans trois domaines: compréhension de l'écrit, mathématiques, sciences naturelles. L'organisation a été le fait de l'OCDE, les décisions importantes étant prises par une conférence des représentants des ministères de l'Éducation de chaque pays ; la partie scientifique et technique a été prise en charge par un consortium de plusieurs laboratoires de recherche, pilotés par un laboratoire australien.

2. PISA, plutôt que d'évaluer l'acquisition de connaissances spécifiques, évalue l'aptitude à réaliser des tâches qui s'inscrivent dans des situations de vie réelle et qui mobilisent une compréhension de concepts fondamentaux.

3. C'est en « compréhension de l'écrit » que l'évaluation a été la plus complète. Les écrits à comprendre étaient de natures diverses (textes de plusieurs sortes, mais aussi graphiques) et plusieurs dimensions de la compréhension étaient investiguées: trouver une information, comprendre globalement l'écrit, l'interpréter, réfléchir sur son contenu.
4. Les épreuves comprenaient pour moitié des items appelant des réponses à choix multiples et pour moitié des items appelant des réponses construites (Ex. : " Lequel de ces deux textes - qui argumentent en sens inverse - approuvez-vous le plus ? Expliquez votre réponse en utilisant vos propres mots pour vous référer aux deux textes »).

5. Les items des épreuves ont été proposés par les pays eux-mêmes, puis ont été revus par des spécialistes de la discipline et des experts en évaluation, puis soumis en retour à l'approbation de chaque pays. Pour chaque langue, les épreuves ont été traduites de deux versions-sources, l'une en français et l'autre en anglais, et ces traductions travaillées jusqu'à ce qu'elles coïncident.

6. Les élèves ont, en outre, passé une demi-heure à répondre à un questionnaire sur leur environnement familial et scolaire.

7. En 2003, puis 2006, les élèves de 15 ans seront à nouveau évalués, avec les maths pour dominante en 2003 et les sciences en 2006. 


\section{La question des biais culturels dans PISA}

Un pays peut être « avantagé » dans une évaluation internationale par de multiples facteurs, dont certains sont évoqués dans le corps du texte: le redoublement si la population est définie par le niveau scolaire et non pas par l'âge, la définition de la populationmère, l'échantillonnage, le degré d'intégration des élèves les plus faibles (y compris les handicapés, par exemple), la gestion du cas des élèves absents le jour de l'épreuve, etc.

Toutefois, ce sont les «biais culturels" qui font couler le plus d'encre, surtout dans notre pays. Ceuxci peuvent tenir à la culture générale du pays ou à ce qu'on pourrait appeler sa culture scolaire, c'est-àdire le contenu ou « l'esprit » des programmes (curriculum officiel) et de ce que les enseignants en enseignent réellement dans leur classe (curriculum réel). II est admis généralement que la conception et la réalisation de PISA ont réduit ces biais au maximum de ce que les techniques actuelles autorisent en la matière.

Mais les biais culturels qui demeurent sont-ils de nature à fausser l'appréhension des écarts de compétence entre pays, tels que ceux que nous mobilisons dans cet article ? La réponse nous paraît négative. Certains faits, sans le prouver, le laissent penser: les pays "performants " appartiennent à des aires culturelles très différentes; des pays de même culture ont des performances sensiblement différentes (par exemple, les USA et les pays du Commonwealth, les Suisses allemands et les Allemands eux-mêmes) ; des pays de même langue ont des performances différentes (les Québécois ont des performances bien meilleures que les Français, les Anglais que les Américains).

Par ailleurs, une étude réalisée dans le cadre de PISA plaide dans le même sens. Pour tester l'ampleur de ces biais culturels en compréhension de l'écrit, il a été proposé aux pays de classer les exercices (items) de l'épreuve commune sur une échelle de 0 ("Cet item ne convient pas pour évaluer les élèves de notre pays ") à 4 ("Cet item convient particulièrement bien pour évaluer les élèves de notre pays »). Dix-huit pays se sont prêtés à l'exercice, dont la France. Puis, le score moyen des pays a été calculé par les experts de PISA en prenant en compte, non pas l'ensemble des items, mais seulement ceux que les experts de chaque pays avaient classés « 4 »(OECD, 2002b). En classant les pays selon le pourcentage moyen d'items corrects, la France est $14^{e}$ sur 32 si l'on se fonde sur l'ensemble des items de PISA et $12^{\mathrm{e}}$ si l'on utilise, pour calculer la performance de tous les pays, seulement les résultats obtenus par leurs élèves aux items classés « 4 » par la France. Il y a donc un biais si l'on veut absolument appeler "biais " l'écart qui existe entre le classement qui résulte des items décidés par la collaboration entre les experts de tous les pays et celui qui résulterait de l'imposition de nos propres critères à tous les autres pays - mais de peu de conséquence.

De plus, l'origine culturelle de ce biais, semble douteuse : en utilisant les items classés 4 par des pays "de culture anglo-saxonne ", le classement de la France se situe entre $11^{\mathrm{e}}$, avec les items privilégiés par la Nouvelle Zélande, et $15^{\mathrm{e}}$, avec les items privilégiés par l'Australie. En utilisant des items classés « 4 » par des pays latins, il oscille entre $11^{\mathrm{e}}$ (Grèce) et $16^{\mathrm{e}}$ (Italie). À quoi on peut ajouter que, en utilisant les items «français » plutôt que l'ensemble des items pour classer les États-Unis et le Royaume-Uni, on ne change pas la place des États-Unis et on en fait gagner une au Royaume-Uni. On voit donc que le classement de la France, comme celui des autres pays, est peu sensible à l'aire culturelle du pays pris pour référence. 


\section{Variables des questionnaires PISA reliées à l'efficacité de l'apprentissage}

\section{Aide et stimulation par l'environnement familial}

\section{Communication culturelle avec les parents}

Avec quelle fréquence :

- discutes-tu avec tes parents de questions politiques ou sociales?

- discutes-tu sur des livres, des films ou des programmes de télé ?

- écoutes-tu de la musique classique avec tes parents?

\section{Soutien familial}

À quelle fréquence les personnes suivantes vous aident-elles pour votre travail scolaire à la maison : mère, père, frères ou sœurs, grands parents, d'autres connaissances, des amis de vos parents ?

\section{Temps de travail à la maison}

En moyenne, combien de temps passes-tu chaque semaine à travailler ou à étudier pour le français ? pour les mathématiques? pour les sciences?

\section{Richesse des ressources éducatives} à la maison
À la maison, disposez-vous :
- d'un dictionnaire,
- d'un endroit calme pour étudier,
- d'un bureau pour étudier,
- de manuels scolaires,
- d'une calculatrice.

\section{Efficacité de l'enseignement}

\section{Encouragement (Pression à la réussite)}

Dans les cours de français :

- le professeur demande aux élèves de travailler beaucoup ;
- dit aux élèves qu'ils pourraient mieux faire ;

- est mécontent lorsque les élèves remettent un travail mal fait ;

- les élèves ont beaucoup à étudier.

\section{Soutien par les enseignants}

Dans les cours de français :

- le professeur s'intéresse aux progrès de chaque élève ;

- donne aux élèves l'occasion d'exprimer leur opinion ;

- aide les élèves dans leur apprentissage ;

- continue à expliquer jusqu'à ce que les élèves aient compris ;

- s'investit beaucoup pour aider les élèves ;

- aide les élèves dans leur travail.

(jamais, parfois, à la plupart des cours, à chaque cours)

\section{(Bonnes) Relations entre les élèves et les enseignants}

Dans mon établissement :

- les élèves s'entendent bien avec le professeur ;

- les professeurs s'intéressent au bien-être de leurs élèves ;

- sont réellement à l'écoute de ce que j'ai à dire ;

- si j'ai besoin d'aide supplémentaire, mes professeurs me l'apportent ;

- mes professeurs me traitent équitablement.

\section{Discipline et engagement des élèves}

\section{(Mauvais) Climat disciplinaire selon les élèves}

Dans les cours de français :

- le professeur doit attendre un long moment avant que les élèves ne se calment ;

- il y a du bruit et de l'agitation ;

- les élèves ne peuvent pas bien travailler; 
- les élèves n'écoutent pas ce que dit le professeur ;

- les élèves ne commencent à travailler que bien longtemps après le début du cours.

Au début du cours, plus de cinq minutes se passent sans qu'on ne fasse rien.

Cet indicateur a été inversé pour obtenir la qualité de la discipline.

\section{(Mauvais) Climat disciplinaire selon le chef d'établissement}

L'apprentissage des élèves est gêné par l'absentéisme, les élèves qui perturbent le cours, les élèves qui sautent des cours, le manque de respect pour les professeurs, l'usage d'alcool et de drogue par les élèves, le fait que des élèves intimident ou maltraitent d'autres élèves.

Cet indicateur a été inversé pour obtenir la qualité de la discipline. 